\title{
Botulinum Toxin-A for the Treatment of Voiding Dysfunction: Our Initial Experience
}

\author{
Sinharib Citgez, Bulent Onal, Fetullah Gevher, Cagatay Dogan, Omer Kurt, Oktay Demirkesen, \\ Bulent Cetinel ${ }^{*}$ \\ Department of Urology, Cerrahpasa Medical Faculty, University of Istanbul, Istanbul, Turkey \\ Email: *bulent.cetinel@hotmail.com
}

Received March 14, 2012; revised March 26, 2012; accepted April 10, 2012

\begin{abstract}
Objective: Evaluation of Botulinum Toxin-A (BTX-A) as an alternative treatment option in patients whom previous treatments were failed. Methods: Between March 2005 and September 2006, a total 19 patients; 16 patients with overactive bladder (5 neurologic, 11 idiopathic), 2 with nocturnal enuresis, 1 with interstitial cystitis, intravesical BTX-A injection was applied. Except one patient, 18 patients with a median age $46(20-80)$ were registered to the study. Patients were examined at postoperative 3 weeks and 6 months with ICIQ-SF (International Consultation on Incontinence Questionnaire-Short Form) and satisfaction forms used in our clinic. Results: The median follow-up time was 16 months $(6-18)$. When evaluated according to diagnosis, symptoms were improved $100 \%$ in neurologic overactive bladder $(5 / 5), 80 \%$ in idiopathic overactivity $(8 / 10), 50 \%$ in nocturnal enuresis $(1 / 2)$. In one patient with interstitial cystitis there was no improvement. Median efficiency time of BTX-A treatment was 6 months $(1-17)$. At 3 weeks and 6 months after the treatment, ICIQ-SF score was decreased to 3.6 and 7.5 respectively from preoperative level of 18.7. Quality of life scores at preoperative, 3 week and 6 months were 9.7, 2.7 and 4.3 respectively. Except 3 patients with overactive neurologic bladder because of spinal cord injury, symptoms of patients with pollacuria and nocturia decreased at 3. week. Even if these rates increased at 6 months they still were significantly less than preoperative status. The spinal cord injured 3 patients had clean intermittent catheterization per 1 hour and had urinary incontinence between the catheterizations preoperatively, however at 3 weeks and 6 months the intervals of catheterisations was 4 hours and 3 hours respectively and also the urinary incontinance between the catheterisations improved. At patient satisfactions, $78 \%$ of patients declared that they were pleased and underwent the procedure again and will recommend it to their relatives with similar problems. One patient had a complication as tansient weakness on her legs. Conclusion: BTX-A injections provide an alternative treatment before surgery at overactive bladder especially for those who doesn't response to medical treatment or cannot use antimuscarinics because of their side effects. Further studies with a greater number of patients are needed for determining the effectiveness of BTX-A on patients with voiding dysfunctions.
\end{abstract}

Keywords: Botulinum Toksin-A; Overactive Bladder; Voiding Dysfunction

\section{Introduction}

Botulinum toxin (BTX) was described first by Van Ermengem in 1897 [1]. Botulinum is known as the most potent biological toxin and has been increasingly used as an effective treatment method in numerous diseases.

Botulinum toxin has 7 different serotypes called A, B, $\mathrm{C}, \mathrm{D}, \mathrm{E}, \mathrm{F}$ and $\mathrm{G}[2]$. However, only $\mathrm{A}$ and $\mathrm{B}$ types are used for treatment purposes. Demonstrating of BTX to produce neuromuscular blockade has been a rationale for its clinical practicability [3]. However, first usage of this toxin in the treatment was for resolving of strabismus in human, and the results were published in 1980 [4]. Botulinum toxin shows its muscle relaxant effect by transiently inhibiting the release of acetylcholine in

"Corresponding author. presynaptic cholinergic junction. The clinical effect of Botulinum toxin is transient and depends on the dose. BTX has been given efficient and reliable results so far with reversible effects, which increase the medical area of use for this toxin. It is used for muscle spasm and resultant many head and neck diseases with a painful course. These diseases include blepharospasm, facial dyskinesias, spastic dysphonia, oromandibular dystonia and spasmodic torticollis. In addition, BTX is also used for hyperhidrosis, achalasia, focal spasticity and resolving of wrinkles.

In urological area, BTX has been used in detrusor sphincter dyssynergia (DSD) [5], urinary dysfunction [6], neurogenic detrusor overactivity (NDO) [7] or idiopathic/ non-neurogenic detrusor overactivity (NNDO) [8], chronic prostatitis [9], chronic pelvic pain syndrome [10] and 
recurrent urethral strictures [11]. Patients must be selected carefully since BTX injection is an expensive operation, requiring general anesthesia. In this study, we aimed to evaluate effectiveness of BTX-A injection that we performed in the patients with urinary dysfunction and to evaluate the patient satisfaction.

\section{Material and Methods}

BTX-A injection was retrospectively studied in a total of 19 patients; 16 had overactive bladder ( 5 neurogenic, 11 idiopathic), 2 nocturnal enuresis and 1 interstitial cystitis between March 2005 and September 2006. Except one patient who was lost in the follow-up, 18 patients with a median age of 46 years (range 20 - 80) were included in the study. This study was conducted on the patients who had previously received antimuscarinic therapy at least for 6 months, which had failed or not provide a sufficient benefit with persistent complaints. Following the detailed history and examination, patients were evaluated with 3-day urinary diary, full urinalysis, urine culture, ICIQ-SF (International Consultation on Incontinence QuestionnaireShort Form), urinary system ultrasonography, routine biochemical tests and urodynamics. In the urodynamic measurement; urodynamic bladder capacity, first desire to void, strong desire to void, detrusor pressure (Pdet) and residual urine values were analyzed. Standards of the Internationsl Continence Society (ICS) were complied in urodynamic definitions [12].

ICIQ-SF forms were filled preoperatively by the patients. The first three questions helped us to understand the severity of irritative symptoms, and the next 3 questions were asked to understand the complaints related to urinary incontinence. Finally, we studied the extend of these complaints in the daily activities with the life quality score giving information on the quality of life with a scale between 0 and 10 .

Patients with overactive bladder consisted of 5 patients with NDO including 3 had medulla spinalis injuries, 1 had suffered from poliomyelitis and 1 multiple sclerosis (MS) patient, and 11 patients having NNDO. Two of three medulla spinalis injuries were due to a traffic accident (T4-5 injury) and 1 had caused by falling from height (T8 injury) 8 years ago (Table 1). Except the MS patient, clean intermittent catheterization (CIC) durations were shorter (CIC intervals; 0.5 - 1 hour) and there was a complaint of incontinence between the CICs in other patients with NDO and applied CIC. Of the patients with NNDO, 9 had urge urinary incontinence and 2 patients had hypocompliant, hypersensitive bladder urodynamic signs with irritative complaints. Two of the 3 remaining patients had detrusor overactivity (DO) detected on urodynamics with nocturnal incontinence and received a failed antimuscarinic therapy, and 1 had interstitial cystitis.

After accessing to the bladder using 22 Fr rigid cystoscope under the spinal sedoanalgesia, patients were

Table 1. Patients demographics.

\begin{tabular}{|c|c|c|c|c|c|}
\hline Patient No & Gender & Age & Status & Urodynamic diagnosis & Dose applied (Unit) \\
\hline 1 & $\mathrm{~F}$ & 43 & Medulla Spinalis Injury & Neurogenic DO & 300 \\
\hline 3 & $\mathrm{~F}$ & 38 & Medulla Spinalis Injury & Hypersensitive + Neurogenic DO & 300 \\
\hline 4 & $\mathrm{~F}$ & 25 & Multiple Sclerosis & Hypersensitive + Neurogenic DO & 200 \\
\hline 5 & $\mathrm{~F}$ & 31 & Suffered from poliomyelitis & Hypersensitive + Neurogenic DO & 300 \\
\hline 6 & $\mathrm{~F}$ & 23 & Nocturnal Enuresis & DO & 200 \\
\hline 7 & M & 20 & Nocturnal Enuresis & $\mathrm{DO}$ & 200 \\
\hline 8 & $\mathrm{~F}$ & 39 & Interstisial Cystitis & Hypocompliant + Hypersensitive & 200 \\
\hline 9 & $\mathrm{~F}$ & 46 & Urged urinary incontinence + pollakiuria & Hypocompliant + Hypersensitive & 200 \\
\hline 11 & $\mathrm{~F}$ & 63 & Urged urinary incontinence + pollakiuria & DO & 200 \\
\hline 12 & $\mathrm{~F}$ & 60 & Urged urinary incontinence + pollakiuria & DO & 200 \\
\hline 13 & $\mathrm{~F}$ & 63 & Pollakiuria +Nocturia & Hypocompliant & 200 \\
\hline 14 & M & 51 & Pollakiuria + Nocturia & Hypocompliant + Hypersensitive & 200 \\
\hline 15 & $\mathrm{~F}$ & 74 & Urged urinary incontinence & DO & 200 \\
\hline 16 & $\mathrm{~F}$ & 40 & Urged urinary incontinence + pollakiuria & DO & 200 \\
\hline 17 & $\mathrm{~F}$ & 80 & Urged urinary incontinence + pollakiuria & Hypersensitive + DO & 200 \\
\hline 18 & $\mathrm{~F}$ & 58 & Mixed Incontinence & DO & 200 \\
\hline
\end{tabular}


injected with previously diluted botulinum toxin-A from the detrusor muscle. The dilution was made with 100 unit botox/10cc isotonic. The injection was made into posterior and lateral walls and to the trigone region. Four patients with NDO were administered 300 units to 30 different points, while $1 \mathrm{MS}$ patient and remaining 14 patients with NNDO received 200 units to 20 different points as described previously [7].

Follow-up of the patients was carried out through ICIQ-SF and satisfaction forms (Questionnaire-1) used in our clinics at postoperative 3 rd weeks and 6 th months. In this interrogation, their responses were received about when the complaints began again, whether the patient was satisfied with this therapy, would the patients seek this therapy again, and would the patients recommend this method to their relatives having the same condition. In addition, questions of "what was the preoperative CIC interval and do you have urinary incontinence between the CICs?" were asked to our 5 patients with NDO.

\section{Results}

Median follow-up duration of 18 patients was 16 months (range 6 - 18). When the results were evaluated according to the diagnoses; relief from the symptoms was achieved in neurogenic overactivity by $100 \%(5 / 5)$, idiopathic overactivity by $80 \%(8 / 10)$ and nocturnal enuresis by $50 \%(1 / 2)$, while no relief was obtained in our 1 patient with interstitial cystitis.

Median efficient time of BTX-A was 6 months (range 1 - 17). At 3 weeks and 6 months after the treatment, ICIQ-SF score was decreased to 3.6 and 7.5 respectively from preoperative level of $18.7(\mathrm{p}<0.05)$. Quality of life scores at preoperative, 3 week and 6 months were 9.7, 2.7 and 4.3 respectively. Except 3 patients with overactive neurologic bladder because of spinal cord injury, symptoms of patients with pollakiuria and nocturia decreased at 3 week Even if these rates increased at 6 months they still were significantly less than preoperative status. (Table 2). The spinal cord injured 3 patients had CIC per 1 hour and had urinary incontinence between the cathe- terizations preoperatively; however, at 3 weeks and 6 months the intervals of catheterizations was 4 hours and 3 hours respectively and also the urinary incontinence between the catheterizations improved.

At patient satisfactions, $78 \%$ of patients declared that they were pleased and underwent the procedure again and will recommend it to their relatives with similar problems.

Hypersensitive DO was identified on urodynamics of one MS patient with urged incontinence. The patient with persistent complaints despite antimuscarinic therapy continuing for 1 year had ICIQ-SF values of 20, 0 and 6 at preoperative, $3 \mathrm{rd}$ week and 6th month respectively, while frequency of diurnal voiding was 12,4 and 5, and nocturnal voiding frequency was 4, 0 and 1 times. In the follow-up of the patient, the complaints were resolved, but the patient made CIC one at 4 hours since he was not able to full continence. The patient was under follow-up for 7 months, and he had not incontinence during this period. While the patient was not able to make CIC before BTX-A injection, he reported that he was satisfied with the therapy despite CIC.

Wilcoxon tests were used to compare the conditions of the patients at preoperative period and postoperative $3 \mathrm{rd}$ weeks and 6th months.

\section{Discussion}

This study showed that BTX-A injection was satisfying in $78 \%$ of the patients. Pollakiuria, and nocturia were decreased in all the patients. This result was consistent with the studies in the literature which reported decreasing in the frequency of voiding and incontinence episodes with BTX-A therapy $[8,13,14]$.

We performed BTX-A injection under general anesthesia and spinal anesthesia. There are also some authors who performed this operation under local anesthesia with a flexible cystoscope $[14,15]$. These patients stated that they felt anxious during the operation, but they could undergo the same procedure in case of the recurrence of

Table 2. Datas of pre- and postoperative BTX-A injection.

\begin{tabular}{lllll}
\hline & Preoperative & Postoperative 3 week & Postoperative 6 month & p \\
\hline Mean ICIQ-SF score (range) & 18.7 & 3.6 & 7.5 & $0.001^{* *}$ \\
& $(16-20)$ & $(0-20)$ & $(0-20)$ & $0.003^{* *}$ \\
& & & & $0.018^{* * *}$ \\
& & & $0.000^{*}$ \\
Mean Quality of Life Complaint Score (range) & 9.7 & $(0-10)$ & $(0-10)$ & $0.002^{* *}$ \\
& $(8-10)$ & 6.5 & 7.3 & $0.007^{* * *}$ \\
& 13.7 & $(2-20)$ & $(2-20)$ & $0.000^{*}$ \\
Pollakiuria (median/day) (range) & $(6-25)$ & 1.4 & 2.0 & $0.002^{* *}$ \\
& 4.3 & $(0-10)$ & $(0-10)$ & $0.017^{* * *}$ \\
Nocturia (median/day) (range) & $(1-10)$ & & $0.000^{*}$ \\
& & & & $0.002^{* *}$ \\
\end{tabular}

"Preoperative and postoperative 3 week; ${ }^{* *}$ Preoperative and postoperative 6 month; ${ }^{* *}$ Postoperative 3 week and 6 month. 
their complaints. In another study, BTX-A accompanied by cystoscopy with intravenous sedation was reported with a good patient tolerance [16].

Reason of the trigone not to be injected BTX-A was stated as the paralysis occurring in the trigone might cause to vesicoureteral reflux (VUR) [7]. However, Smith et al. stated that they performed the injection of BTX particularly into the trigone site in NDO $[17,18]$. The authors underlined that they did not look for VUR in their patients after the injection; however, they did not find episodes of pyelonephritis in any patient, and thus they performed injections to the trigone site assuming injections to this region, which is afferent neurons-rich (especially $\mathrm{C}$ fibers) would have more antinociceptive effects [18]. In our practice, all the patients were injected from the trigone regions. We also did not look for VUR in our patients, but no episode of pyelonephritis was found in any of them. We think further studies on this subject are needed.

The dose of BTX-A into the bladder which would show the maximum therapeutic effect, and a minimal side effect is still not known. Schurch et al. are the first applying this procedure on the widest series and they applied BTX-A injection with 300 units [7]. This amount was accepted in numerous subsequent studies and has been successful $[15,19,20]$. Some authors reported they administered 300 units of BTX-A in NDO and NNDO conditions while the others stated they used 200 units in NNDO and 300 in NDO [7,18,21]. We administered BTX-A injection as 300 units in 4 neurogenic patients and 200 units in 14 NNDO patients. Rationale for lower dose in NNDO is stated as refraining from the urine retention resulting in CIC requirement in these patients [21]. No significant residual urine was found in the patients in postoperative follow-up. One MS patient required CIC once at 4 hours after botox injection of 200 units. Chancellor et al. treated 10 NNDO patients with 100 to 300 units of BTX-A and found reduction in frequency of urination in the follow-up in $80 \%$ of the patients [8]. Median efficient time of BTX-A was 6 months (range 1 - 17) in our study. At 3 weeks and 6 months after the treatment, ICIQ-SF score was decreased to 3.6 and 7.5 respectively from preoperative level of 18.7 ( $p<$ 0.05 ). Quality of life scores at preoperative, 3 . week and 6. months were 9.7, 2.7 and 4.3 respectively.

In a multicenter, placebo controlled double-blinded randomize study, Schurch et al. [22] investigated low doses of botox. In that study, 19 patients were administered 200 units and 19 patients 300 units of BTX-AB, while the other 21 patients received placebo. The most importance result of this study was found as the significant reduction in frequency of voiding and incontinence episodes in both groups. Therefore, site, number, technique and dosage of the injection gain importance. Further studies are needed to define the low doses would not require CIC postoperation, while having therapeutic efficacy.

There are few studies related to BTX-A application to relieve the pain in patients with interstitial cystitis (IC). In a series of 110 patients by Smith et al., Botox was applied to the bladder in 4 patients with IC [22]. In that study, BTX-A was stated to be applied in the afferent neuron-rich trigone region as 100 units in the patient with IC and this application was proposed to be considered also in the patients with IC. In a review article, Frenkl and Rackley reported that they applied BTX-A in 10 patients with IC and the results were not positive [21]. In our one patient with IC, we injected 100 of 200 units to the trigone and 100 randomly to the other parts of the bladder, but no improvement was seen in the complaints.

Some side effects were reported with BTX-A injections in urology. Although it is assumed that BTX-A remains in the injected muscle, some studies reported systemic side effects such as hyposthenia and general muscle weakness [21]. Our one patient developed weakness in the legs, although this was not severe enough to prevent walking, and the complaints disappeared after 1 month. However, we could not rule out the possibility of whether this weakness had resulted from the known previous diseases such as vitamin B12 deficiency, gluten enteropathy and ionized calcium deficiency. The patient reported that he had similar complaints preoperation. In our study, $78 \%$ of patients declared that they were pleased and underwent the procedure again and will recommend it to their relatives with similar problems.

There was not incontinence between CICs for 6 months after BTX-A injection in the young patient who had suffered from poliomyelitis, had bilateral paralysis, received antimuscarinic therapy for 8 years and had complaints of urinary incontinence between the catheterizations despite he applied CIC, but later the complaints were repeated. The patient was satisfied compared the preoperation, but he underwent bladder augmentation surgery since he wanted permanent treatment.

In this study, efficient time of BTX-A injection into the bladder was consistent with those were reported in the literature $[7,8]$. Because there were not repeating applications in our study, efficient time and results of treatment are not known in the repeating doses. In a study by Ghalayini et al., similar results with the initial application were obtained in re-injections performed when the efficient was reduced 5 - 8 months after the first operation in the patients with NDO and NNDO [23].

Despite efficient results in NDO and NNDO in which antimuscarinic therapy was received for a long-term, the most important disadvantage of BTX-A application is to be transient. Nevertheless, BTX-A therapy is an important treatment option for the patients who could not give a response to antimuscarinic therapy. In addition, all the 
patients were satisfied postoperatively and they stated that they could receive BTX-A injection again in case of the repeating of complaints.

\section{REFERENCES}

[1] E. Van Ermengem, "A New Anaerobic Bacillus and Its Relation to Botulism," Clinical Infectious Diseases, Vol. 1, No. 4, 1979, pp. 701-709. doi:10.1093/clinids/1.4.701

[2] C. L. Comella, M. Brin and J. Jankovic, "Biochemistry of Botulinum Toksin Type B," Neurology, Vol. 55, No. 12, 2000, pp. 22-28.

[3] A. S. V. Burgen, F. Dickens and L. J. Zatman, "The Action of Botulinum Toxin on the Neuromuscular Junction," Journal of Physiology, Vol. 109, No. 1-2, 1949, pp. 10-24.

[4] A. B. Scott, "Botulinum Toxin Injection into Extraocular Muscles as an Alternative to Strabismus Surgery," Journal of Pediatic Ophtalmology and Strabismus, Vol. 17, No. 10, 1980, pp. 1044-1049.

[5] D. D. Dykstra, A. A. Sidi, A. B. Scott, et al., "Effects of Botulinum A Toxin on Detrusorsphincter Dyssynergia in Spinal Cord İnury Patients," The Journal of Urology, Vol. 139, No. 2, 1988, pp. 919-922.

[6] H. C. Kuo, "Botulinum A Toxin Urethral Injection for the Treatment of Lower Urinary Tract Dysfunction," The Journal of Urology, Vol. 170, No. 5, 2003, pp. 1908-1912. doi:10.1097/01.ju.0000091281.50081.f0

[7] B. Schurch, M. Stohrer, G. Kramer, et al., "Botulinum-A Toxin for Treating Detrusor Hyperreflexia in Spinal Cord Injured Patients: A New Alternative to Anticholinergic Drugs Preliminary Results," The Journal of Urology, Vol. 164, No. 3, 2000, pp. 692-697. doi:10.1016/S0022-5347(05)67283-7

[8] M. Chancellor, M. O'Leary, J. Erickson, et al., "Successful Use of Bladder Botulinum Toxin Injection to Treat Refractory Overactive Bladder," The Journal of Urology, Vol. 169, Suppl. 4, 2003, p. 351.

[9] D. Zermann, M. Ishigooka, J. Schubert, et al., "Perisphincteric Injection of Botulinum Toxin Type A: A Treatment Option for Patients with Chronic Prostatic Pain?" European Urology, Vol. 38, No. 4, 2000, pp. 393-399. doi: $10.1159 / 000020314$

[10] S. K. Jarvis, J. A. Abbott, M. B. Lenart, et al., "Pilot Study of Botulinum Toxin Type A in the Treatment of Chronic Pelvic Pain Associated with Spasm of the Levator Ani Muscles," Australian and New Zealand Journal of Obstetics and Gynaecology, Vol. 44, No. 1, 2004, pp. 4650. doi:10.1111/j.1479-828X.2004.00163.X

[11] M. Khera, T. B. Boone and C. P. Smith., "Botulinum Toxin Type A: A Novel Approach to the Treatment of Recurrent Urethral Strictures," The Journal of Urology, Vol. 172, No. 2, 2004, pp. 574-575. doi:10.1097/01.ju.0000130652.27541.22

[12] P. Abrams, L. Cardozo, M. Fall, et al., "The Standardization of Terminology of Lower Urinary Tract Function: Report from the Standardisation Sub-Committee of the International Continence Society," Neurourology and Urodynamics, Vol. 21, No. 2, 2002, pp. 167-178.

\section{doi:10.1002/nau.10052}

[13] T. M. Kessler, H. Danuser, M. Schumacher, et al., "Botulinum A Toxin Injections into the Detrusor: An Effective Treatment in Idiopathic and Neurogenic Detrusor Overactivity?" Neurourology and Urodynamics, Vol. 24, No. 3, 2005, pp. 231-236. doi:10.1002/nau.20105

[14] R. Popat, A. Apostolidis, V. Kalsi, et al., "Comparison between the Response of Patients with İdiopathic Detrusor Overactivity and Neurogenic Detrusor Overactivity to the First Intradetrusor Injection of Botulinum-A Toxin," The Journal of Urology, Vol. 174, No. 2, 2005; pp. 984-989. doi:10.1097/01.ju.0000169480.43557.31

[15] M. Harper, R. B. Popat, R. Dasgupta, et al., "Minimally İnvasive Technique for Outpatient Local Anaesthetic Administration of Intradetrusor Botulinum Toxin in Intractable Detrusor Overactivity," BJU International, Vol. 92, No. 3, 2003, pp. 325-326. doi:10.1046/j.1464-410X.2003.04312.x

[16] D. E. Rapp, A. Lucioni, E. E. Katz, et al., "Use of Botulinum-A Toxin for the Treatment of Refractory Overactive Bladder Symptoms: An Initial Experience," Urology, Vol. 63, No. 3, 2004, pp. 1071-1075. doi:10.1016/j.urology.2004.01.041

[17] C. P. Smith and M. B. Chancellor, "The Emerging Role of Botulinum Toxin in the Management of Voiding Dysfunction," The Journal of Urology, Vol. 171, No. 6, 2004, pp. 2128-2137. doi:10.1097/01.ju.0000127725.48479.89

[18] C. P. Smith, S. Nishuguchi, M. O'Leary, et al., "Single Institution Experience in 110 Patients with Botilinum A Toxin Injection into Bladder or Urethra," Urology, Vol. 65 , No. 1, 2005, pp. 37-41. doi:10.1016/j.urology.2004.08.016

[19] H. Schulte-Baukloh, T. Michael, B. Sturzebecher, et al., "Botulinum-A Toxin Detrusor İnjection as a Novel Approach in the Treatment of Bladder Spasticity in Children with Neurogenic Bladder," European Urology, Vol. 44, No. 1, 2003, pp. 139-143. doi:10.1016/S0302-2838(03)00136-2

[20] A. Reitz, M. Stohrer, G. Kramer, et al., "European Experience of 200 Cases Treated with Botulinum-A Toxin Injections into the Detrusor Muscle for Urinary Incontinence Due to Neurogenic Detrusor Overactivity," European Urology, Vol. 45, No. 4, 2004, pp. 510-515. doi:10.1016/j.eururo.2003.12.004

[21] T. L. Frenkl and R. R. Rackley, "Injectable Neuromodulatory Agents: Botulinum Toxin Therapy," Urologic Clinics of North America, Vol. 32, No. 1, 2005, pp. 89-99. doi:10.1016/j.ucl.2004.09.005

[22] C. P. Smith, S. Nishuguchi, M. O’Leary, et al., "Single Institution Experience in 110 Patients with Botilinum A Toxin İjection into Bladder or Urethra," Urology, Vol. 65 , No. 1, 2005, pp. 37-41. doi:10.1016/j.urology.2004.08.016

[23] I. F. Ghalayini and M. A. Al-Ghazo, "Intradetrusor Injection of Botulinum-A Toxin in Patients with Idiopathic and Neurogenic Detrusor Overactivity: Urodynamic Outcome and Patient Satisfaction," Neurourology and Urodynamics, Vol. 26, No. 4, 2007, pp. 531-536.

doi:10.1002/nau.20403 